Esta publicación cientifica en formato digital es continuidad de la revista impresa ISSN-Versión Impresa 0798-1406 / ISSN-Versión on line 2542-3185Depósito legal pp
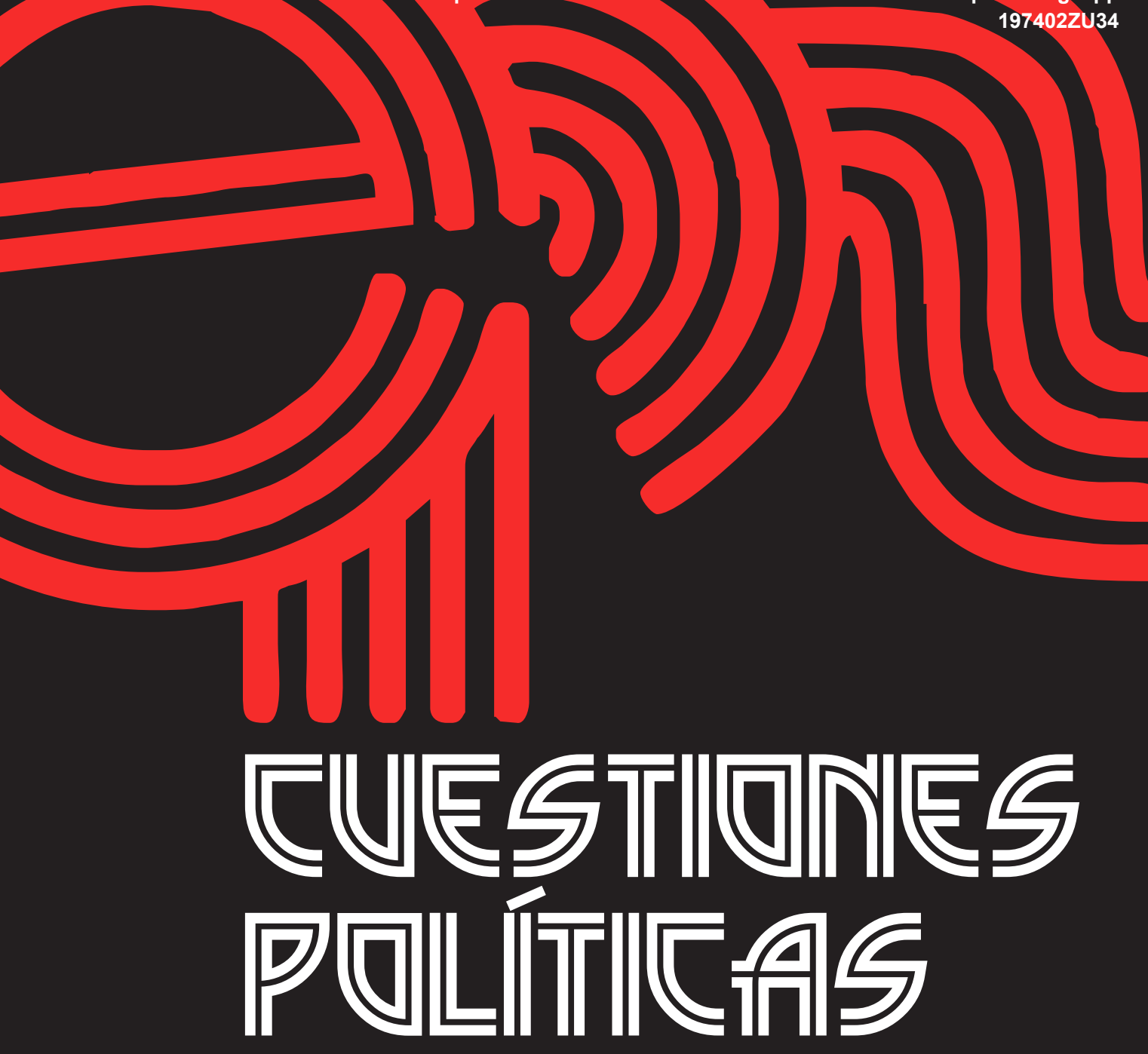

Instituto de Estudios Políticos y Derecho Público "Dr. Humberto J. La Roche" de la Facultad de Ciencias Jurídicas y Políticas de la Universidad del Zulia Maracaibo, Venezuela
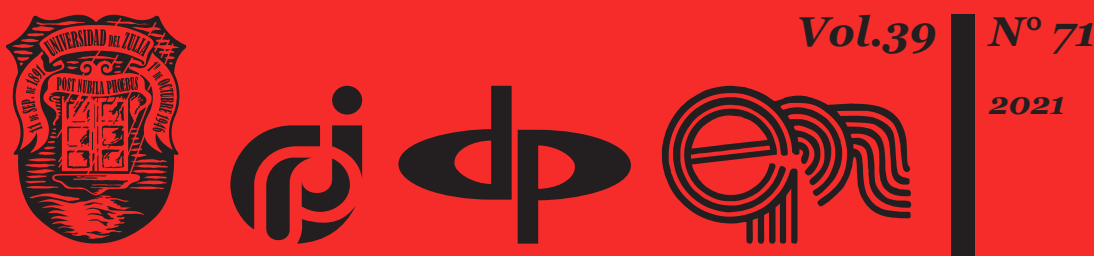


\title{
Deuda pública en México: dinámica e implicaciones de política
}

\author{
DOI: https://doi.org/10.46398/cuestpol.3971.01
}

\author{
Oshiel Martínez Chapa * \\ Jorge Eduardo Salazar Castillo ** \\ Saul Roberto Quispe Aruquipa ***
}

\section{Resumen}

El propósito de este trabajo es analizar los factores que han impulsado la deuda pública en México y sus consecuencias sobre la economía. La hipótesis planteada es que el incremente de la deuda se relaciona con factores tales como la discrecionalidad en el manejo de los recursos públicos, el aval de los recursos petroleros, el costo de los rescates financieros y el creciente gasto social ejercido. La pregunta de investigación fue: ¿Cómo ha evolucionado la deuda pública en el mediano y largo plazo, y qué consecuencias tiene? La metodología utilizada es cualitativa por cuanto analiza los hechos y los documentos, pero también cuantitativa por cuanto echa mano de un modelo de regresión en el cual se emplea una tasa de crecimiento de la variable en cuestión. Los datos proceden de instituciones como el Banco de México, el Banco Mundial, la Secretaría de Hacienda y Crédito Público (SHCP), así como World Population Review. El trabajo concluye destacando la necesidad de que los gobiernos adopten políticas responsables a fin de incidir en el crecimiento y el desarrollo económico, y no que las políticas de austeridad provoquen baja inversión y desempleo en el país.

Palabras clave: deuda pública; gasto público; Estado de Bienestar; política de gobierno; crecimiento económico.

* Profesor del Tecnológico Nacional de México y de la Universidad Autónoma de Tamaulipas. Doctorado en Economía (Instituto Internacional del Derecho y del Estado). Maestro en Economía (Universidad Autónoma de Tamaulipas). Maestro en Administración (Universidad Autónoma de Nuevo León). Profesor del Tecnológico Nacional de México y de la Universidad Autónoma de Tamaulipas. ORCID ID: http://orcid.org/oooo-0oo1-9675-5472.

** Profesor de la Universidad Autónoma de Tamaulipas. Maestro en Administración. Especialización en Computación (Universidad Autónoma de Nuevo León). Ingeniero Agrónomo (Universidad Autónoma Antonio Narro). Profesor de la Universidad Autónoma de Tamaulipas. ORCID ID: http://orcid. org/oooo-0o02-1138-2353.

*** Profesor, investigador independiente y funcionario del Banco Central de Bolivia. Doctorado en Economía (Universidad Autónoma Metropolitana). Maestro en Economía (Universidad Autónoma Metropolitana). Especialización en Econometría (Universidad Nacional Autónoma de México) Especialización en Finanzas (Universidad Nacional Autónoma de México). Investigador independiente y funcionario del Ministerio de Economía y Finanzas Públicas de Bolivia. ORCID ID: https://orcid. org/oooo-0002-3018-2832. 


\title{
Public debt in Mexico: dynamics and policy implications
}

\begin{abstract}
The purpose of this paper is to analyze the factors that have driven the public debt in Mexico and its consequences on the economy. The hypothesis proposed is that the increase in debt is related to factors such as discretion in the management of public resources, the guarantee of oil resources, the cost of financial bailouts and the growing social spending exercised. The research question is: How has public debt evolved in the medium and long term, and what are the consequences? The methodology used is qualitative in that it analyzes the facts and documents, and the second is quantitative in that it uses a regression model in which a growth rate of the variable in question is used. The data come from institutions such as the Bank of Mexico, the World Bank, the Ministry of Finance and Public Credit (SHCP), as well as World Population Review. The paper concludes by highlighting the need for governments to adopt responsible policies in order to influence growth and economic development, and not that austerity policies cause low investment and unemployment in the country.
\end{abstract}

Key words: public debt; public spending; welfare state; government policy; economic growth.

\section{Introducción}

A lo largo de la existencia, las personas solucionan sus asuntos materiales a través de dos vías complementarias, a saber: 1) de los mercados y 2) de la política. En una condición ideal, la competencia en el mercado produce bienestar en las personas en vista de su interés en maximizar su beneficio. Por otra parte, al ser aquél un votante y contribuyente, espera recibir los mejores servicios posibles, toda vez que los partidos políticos compiten entre sí a fin de ofrecer lo mejor de los bienes públicos.

De acuerdo con Dornbusch et al., (2002), la deuda pública implica que el individuo se debe a sí mismo. En ese sentido, se precisa que la deuda no crezca más allá de lo que lo hace la economía. Sin embargo, las deudas tienden a crecer durante las guerras, en los periodos de desempleo - por los bajos ingresos y por las mayores transferencias y subsidios-y aun en épocas de elecciones. Por otro lado, las deudas tienden a disminuir y se contraen en tiempo de paz y cuando se incrementan la producción y las exportaciones.

No son pocos los ciudadanos que se plantean cuestiones fundamentales como: ¿̇por qué los gobernantes gastan sin control en la mayoría de los casos, aun cuando ello trae consecuencias adversas para la sociedad en su conjunto? ¿Por qué se exceden en el gasto aun cuando se tienen límites 
Oshiel Martínez Chapa, Jorge Eduardo Salazar Castillo y Saul Roberto Quispe Aruquipa Deuda pública en México: dinámica e implicaciones de política

establecidos? ¿Por qué no se informa a la ciudadanía sobre las implicaciones de los grandes proyectos de política pública? ¿Cómo hacer que los políticos y funcionarios paguen por sus errores y omisiones en la decisión y aplicación de políticas públicas?

El presente trabajo justifica su abordaje dado el bajo crecimiento económico de la mayoría de los países, incluido México, pues el mismo es menor al crecimiento de la deuda, lo cual ejerce una presión sobre las finanzas públicas fundamentalmente. El primer apartado corresponde a la revisión de la literatura. El segundo aborda la dimensión de la deuda y las implicaciones de política: sus antecedentes y el contexto internacional, así como el contexto de México y la deuda de sus entidades federativas. El tercero se refiere a los métodos y los resultados. El último plantea las conclusiones y las recomendaciones.

\section{Revisión de la literatura}

Según Parkin (2014), la deuda gubernamental es el monto total de dinero que un gobierno ha solicitado a préstamo y es igual a la suma de los déficits en que ha incurrido, menos la suma del superávit presupuestario del pasado. De acuerdo con Ayhan Kose et al (2020), durante el último medio siglo, las economías de mercados emergentes y en desarrollo han vivido episodios recurrentes de acumulación de deuda. Estos episodios nacionales han formado olas globales de deuda, acrecentando la vulnerabilidad de estos países, pues ello tiene consecuencias económicas y financieras dados los aumentos en los costos de refinanciar esas deudas.

El gobierno es un productor de bienes públicos, de modo que la salud, educación y demás van dirigidos al grueso de la sociedad. Por otro lado, las políticas de tipo industrial, comercial, agrícola, de comercio exterior y demás, se orientan a otros segmentos específicos de la sociedad (Samuelson y Nordahus, 2010). Sin embargo, si por algunas razones dichas políticas fallan, la sociedad en su conjunto acaba perdiendo a causa de los costos de oportunidad. En ese sentido, sí importan significativamente las decisiones de la calidad, pertinencia y eficiencia del gasto gubernamental. Los votos y el dinero de los contribuyentes sostienen financieramente a dichos gobiernos y constituyen su razón de ser. Están ahí, justo para el mejoramiento de las condiciones de vida material social.

De acuerdo con Krause (2014), cuando los funcionarios públicos aplican políticas, lo hacen para el conjunto de la sociedad. En este mismo sentido, deben destacarse los asociados a los incentivos e información que identifican a estos actores de la vida política. En el campo de acción pública deben señalarse tareas legitimas propias de los gobiernos, destacándose la producción de bienes públicos, regulación, seguridad, creación de 
leyes, transferencia de recursos, etcétera. Los políticos y funcionarios tienen razones para conocer el impacto de sus decisiones con base en las posibilidades de ser electos, así como de su agenda de cara al futuro de su carrera partidaria. Sus decisiones, sean beneficiosas socialmente o no, entrañan costos normalmente no calculados, aunque estos se dispersan entre los agentes sociales y económicos.

En términos generales, la clase política y gubernamental se halla motivada por su agenda y la del grupo que representa. Para la misma, los votantes no siempre se encuentran del todo informados, o bien, son indiferentes respecto a los asuntos de la vida pública. Aunque en su plataforma política no lo expresan, suelen tener estrechos compromisos con intereses económicos y políticos creados. Por tanto, bien puede establecerse que el grado de intervención pública en la sociedad no es mínima ni enteramente circunstancial, sino más bien planeada. En buena parte de los países se asocia el trabajo político con intereses muy específicos, más que con respecto a sus representados.

Los principios de la ciencia económica, entre los cuales se encuentran la libertad de elegir, la lucha contra la concentración de la riqueza, la eficiencia asignativa, la primacía del interés público, entre otros, en los hechos suelen convertirse en retórica y se dejan de lado. En la literatura económica se señala la conveniencia del libre mercado, la competencia y el gobierno eficiente como socialmente beneficiosos. Sin embargo, no es difícil advertir que en muchos países se aprueben medidas proteccionistas o monopolistas, así como también gastos extraordinarios del gobierno con graves consecuencias.

En buena parte del mundo los gobiernos no se hacen responsables de sus errores de cálculo económico, ni tampoco pagan los costos sociales derivados de políticas dirigidas a beneficiar a funcionarios y a ciertos empresarios, aun cuando estas lesiones el interés público y sean costosas. Así, los costos de los fracasos se relacionan con la adopción de políticas tales como regulaciones excesivas o laxas, esquemas de privatizaciones, expropiaciones, acciones conducentes al déficit, rescates financieros, inflación, pérdida de competitividad de sectores, o inclusive, quiebras con consecuencias de desempleo y demás crisis que recaen en el grueso de la población.

Dentro del ámbito de su competencia, el Estado adopta, entre otras, la política fiscal, relacionada con los gastos e ingresos que ejerce, para lo cual requiere presupuestos -idealmente en equilibrio a largo plazo- . La economía debiera funcionar sosteniblemente, haciendo participar a los diversos agentes económicos de acuerdo con su capacidad de ingreso y beneficio en aras de alcanzarse mayores niveles de bienestar (Blanchard, 2017). 
De acuerdo con Parkin (2014), en el pasado las funciones del Estado eran menores y se limitaban a la inversión en infraestructura y a la provisión de bienes públicos como defensa, seguridad, administración de justicia, creación y adecuación de leyes y normas, entre otras. Sin embargo, con el advenimiento de la Gran Depresión en Estados Unidos (EU) vino también la participación creciente del Estado de Bienestar. A este fenómeno siguió una inercia de expansión en diferentes magnitudes entre los países. En lo general, ello provocó que la corriente monetarista y la Escuela Austriaca se pronunciaran por detener el crecimiento del gasto público.

En el ejercicio del gasto público, sea discrecional o no, hay grandes ganadores. Hay evidencia de ganancias extraordinarias entre agentes privados que invierten en proyectos ambiciosos. Desafortunadamente, se observa en muchos casos que dicho gasto suele ser excesivo, especialmente en las compras a sobreprecio, adquisición de bienes de mala calidad, contratación de servicios innecesarios, entre otras acciones. No se trata solo de los malos manejos, pues hay evidencia de ganancias extraordinarias entre segmentos privados ligados estrechamente a la clase política. Esto pone de manifiesto que el problema que enfrentan las burocracias o quienes emprenden la acción colectiva es que no está del todo clara la dimensión de su ámbito de actuación: o se rigen por decisiones democráticas o por decisión judicial o, bien, por decreto autoritario.

Por otro lado, también los gobiernos tienen una agenda social, en la cual se halla la base electoral considerable. A la misma hay que atender con programas de asistencia. Para Buchanan (2000), una vez que el Estado interviene activamente, beneficiando a los que considera como grupos vulnerables -campesinos, madres solteras, viudas, pobres, etcétera-, contribuye a distorsionar al ideal de igualdad. El autor lo cuestiona por el hecho de provocar un elevado nivel de deuda, déficit y demás formas de intervencionismo, incapaces de sustituir la falta de verdadera democracia y de justicia distributiva.

\section{Dimensión de la deuda e implicaciones de política: antecedentes y el contexto internacional, el contexto de México y el caso de la deuda de las entidades federativas}

En este apartado se abordan tres dimensiones de la deuda pública y sus implicaciones de política, las cuales se describen a continuación.

\subsection{Antecedentes y contexto internacional}

En el pasado, las monarquías y los gobiernos disponían de programas de atención a la población en general, una significativa parte de la cual era pobre. Sin embargo, con el paso de tiempo fueron estructurando la ayuda 
y atención a la sociedad en desventaja. Así fue hasta que sobrevino el llamado Estado de Bienestar. Ante este, una parte de la sociedad consideró indispensable la implementación de este tipo de políticas. Otra parte de la sociedad la consideró clientelar y sin efectividad para superar la pobreza y la brecha social. Según Ropke (1960), las políticas de gasto social deben ir más allá de los esquemas asistencialistas o populistas.

La cuestión es que los subsidios otorgados a cualquier grupo de la sociedad sean pobres o ricos, constituyen una deuda del mañana. Estos políticos oportunistas hipotecan el futuro de un país trasladando deuda a las generaciones venideras. Las políticas intervencionistas, entre las cuales figuran los subsidios generalizados y gasto extraordinario, derivan en inflación crónica, elevado nivel de déficit, deuda y demás costos económicos que acaban restringiendo las libertades individuales y que comprometen seriamente el futuro de las sociedades.

Friedman (1989) sostiene la existencia de un impacto intelectual y político en las ideas de los economistas clásicos como Adam Smith, Jeremy Bentham, David Ricardo y John Stuart Mill, entre otros. Como resultado de ello estas ideas se hicieron extensivas en EU y Gran Bretaña, observándose cómo las barreras arancelarias tendieron a decrecer. En Japón en poco tiempo se advirtió un avance espectacular en el crecimiento de las libertades sociales, asi como en los ámbitos políticos y económicos. Sin embargo, una vez que concluyó la Segunda Guerra Mundial y sobrevino la dictadura militar, la economía de Japón comenzó a estancarse. Los autores sostienen que prácticamente no hubo conflictos mayores entre 1815 y 1914, es decir, casi un siglo de laissez faire con políticas de intervención modesta.

Por su parte, Dicey (2008) sostiene que las ideas del socialismo fabiano se establecieron en favor de colectivismo, lo que permitió una mayor intervención del Estado, destruyéndose así, en muchos casos, la iniciativa y esfuerzo propios. A pesar del avance de las ideas referidas, algunos economistas de la Escuela Austriaca - Frederich von Hayek y Ludwig von Mises-y de Chicago - Frank H. Knight y Jacob Viner-, así como de Gran Bretaña - Lionel Robbins y G. R. Chesterton-, comenzaron a defender las ideas de limitar el tamaño del Estado y sus costosas implicaciones. Defendieron también las ideas de libre mercado. Ello ocurrió gracias al parteaguas que fue la obra de Hayek, Camino de servidumbre de 1944.

Por regla general, los gobiernos de la posguerra adoptaron políticas keynesianas que se orientaron fundamentalmente a recuperar la economía a través de la generación de empleos y el aumento de ingreso. El gasto público comenzó a aplicarse y crecieron los subsidios a empresas en malas condiciones con intenciones proteccionistas, así como los apoyos a desempleados, familias pobres y, en general, a aquellos que no podían valerse por sí mismos. Como es de esperarse, ello significó elevados costos de manutención del sector público (Heilbroner y Milberg, 1999). En ese tenor, 
el gasto público requiere de recursos económicos para su sostenimiento, lo cual se da a partir de medidas como el incremento de impuestos, aumento de deuda, venta y expropiación de activos, cobro extraordinario de bienes públicos, emisión de moneda, confiscación, por mencionar algunos.

Ubicándose en el contexto de la historia reciente debe señalarse que, para el caso específico de la deuda contraída en América Latina, al decir de Méndez y Morales (2000: 69) la situación es la siguiente:

La deuda de los paises latinoamericanos se ha convertido en un factor depresivo y caótico que subordina las políticas nacionales a factores exógenos incontrolables. El dilema que confrontan es dirimir si son o no naciones soberanas capaces de decidir su propio destino. Igualmente, cabe señalar que el proceso ortodoxo de ajuste que se ha instrumentado carece de elementos esenciales de equidad que lo justifiquen.

Existen factores que han contribuido a la inercia de deuda a nivel mundial. Según se observa en la gráfica 1. los fuertes gastos públicos derivados de eventos como el crecimiento del Estado de bienestar; las experiencias de la primera y segunda guerras mundiales; la posguerra y subsecuente carrera armamentista, la irrupción de altas tasas de inflación de los años setenta, además de factores como el prolongado estancamiento de la economía mundial, han impulsado el crecimiento del déficit y la deuda, obligando a recurrir al financiamiento con más impuestos y cargos. En los hechos, esto está implicando menores ingresos disponibles para buena parte de las familias. No es exagerado, por tanto, deducir las causas de la expansión del referido gasto, pues sí hay grandes receptores de sus rentas: empresarios ligados a políticos y funcionarios, por una parte, y los miembros de rentas o ingresos bajos, por la otra. 


\section{Gráfica 1: Patrones históricos de deuda del gubernamental internacional como porcentaje del PIB}

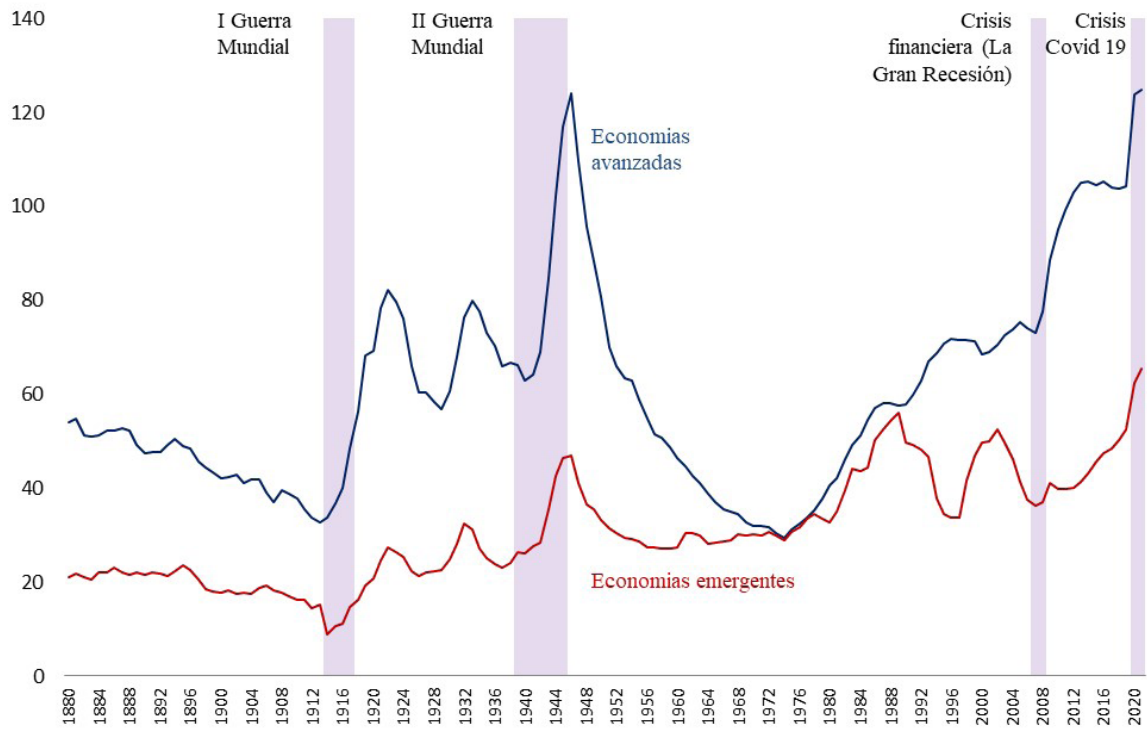

Fuente: Fondo Monetario Internacional (Historical Public Debt Database) y Maddison Database Project. https://www.imf.org/en/Publications/FM/Issues/2020/o9/30/october2020-fiscal-monitor. Nota: La serie de deuda pública agregada a PIB de las economías avanzadas y de los mercados emergentes se basa en una muestra constante de 25 y 27 países, respectivamente, ponderados por el PIB en términos de paridad de poder adquisitivo.

El cuadro No. 1 muestra la deuda como porcentaje del Producto Interno Bruto (PIB) de una lista de países, entre ellos México. En buena medida, la presente deuda contraída es derivada de los rescates financieros acumulados hasta ahora. Hoy constituyen una preocupación respecto a la viabilidad financiera de estos países en el mediano y largo plazo. El principal y el servicio de la deuda entrañan elevados costos financieros, por lo que eventualmente los gobiernos suelen adoptar políticas recesivas con sacrificios para la mayoría de los ciudadanos. La cuestión es que aun en las naciones con mayores niveles de desarrollo y bienestar social existe preocupación dados los niveles extraordinarios de deuda. El entorno internacional adverso se presenta para México como una camisa de fuerza para crecer. Después de la gran recesión de 2008-2009 la recuperación ha sido lenta. 
Oshiel Martínez Chapa, Jorge Eduardo Salazar Castillo y Saul Roberto Quispe Aruquipa Deuda pública en México: dinámica e implicaciones de política

\section{Cuadro No. 1 Deuda como porcentaje del PIB entre países seleccionados}

\begin{tabular}{|l|c|c|c|c|}
\hline Países & 1990 & 2021 & Tasa de variación & $\begin{array}{c}\text { Deuda per cápita } \\
\text { en dólares } \\
28,000\end{array}$ \\
\hline Japón & 52.9 & 237.5 & $349 \%$ & 39,200 \\
\hline $\begin{array}{l}\text { Estalia } \\
\text { Unidos }\end{array}$ & 71.3 & 133.4 & $87 \%$ & 57,300 \\
\hline Chipre & 86.9 & 106.7 & $161 \%$ & 97,200 \\
\hline Gran Bretaña & 30.5 & 85.7 & $181 \%$ & 48,500 \\
\hline India & 50.8 & 69 & $36 \%$ & 340 \\
\hline Israel & 138.5 & 59.5 & $-57 \%$ & 10,700 \\
\hline México & 40.6 & 54.1 & $33 \%$ & 3,200 \\
\hline Dinamarca & 62.2 & 33.6 & $-46 \%$ & 85,700 \\
\hline Suiza & 12.9 & 19.7 & $53 \%$ & 205,000 \\
\hline
\end{tabular}

Fuentes: Cálculos de los autores con base en World Population Review (s/f).

El aumento del gasto público afecta a la viabilidad financiera de las entidades públicas en el mediano y largo plazos. El creciente aumento del techo de la deuda en países como EU, algunos de Europa, Japón y demás está propiciando riesgos mayores de quiebres en la economía real. En el pasado y en el presente las políticas oportunistas e irresponsables de gasto extraordinario han sido denunciadas por algunos sectores de la sociedad, entre ellos el académico. Este tipo de medidas comprometen el futuro viable de millones de personas y significan esquemas de austeridad prolongada. Una parte importante de los ingresos fiscales seguirá canalizándose a pagos del servicio de la deuda a instituciones nacionales y extranjeras, lo cual significa menor ahorro e inversión disponible.

\subsection{El contexto de México}

De acuerdo con la Secretaria de Hacienda y Crédito Público (2020), las obligaciones contratadas por el gobierno federal se componen de los siguientes conceptos: préstamos bancarios, emisiones de valores gubernamentales, deuda con organismos financieros internacionales, bonos del Instituto de Seguridad y Servicios Sociales de los Trabajadores del Estado (ISSSTE) - por la implementación de la nueva Ley del ISSSTEy cuentas relacionadas con la seguridad social, bonos de pensiones - 
Petróleos Mexicanos (Pemex) y Comisión Federal de Electricidad (CFE)-, entre otros. En lo que respecta al endeudamiento del gobierno federal, empresas productivas del Estado y banca de desarrollo, se compone por deuda contratada por entes como el propio gobierno federal, empresas productivas del Estado - Pemex y CFE- y banca de desarrollo - Banco Nacional de Obras y Servicios Públicos (Banobras), Sociedad Hipotecaria Federal (SHF), Banco Nacional de Comercio Exterior (Bancomext), Nacional Financiera (Nafin) y Banco del Bienestar-

Por otro lado, instituciones como la Organización para la Cooperación y el Desarrollo Económico (2009) señalan la necesidad de tener un gobierno más pequeño, descentralizado, promotor del desarrollo inclusivo, proactivo y capaz de atender los más sentidos problemas sociales. Además de los crecientes costos de la deuda pública de México, también merecen señalarse la inercia del gasto asociado a la relación clientelar y su manejo discrecional creados desde hace décadas entre agentes públicos y privados. He aquí los más evidentes:

a) Los grupos de presión que abogan por más recursos o partidas como los partidos políticos; las grandes cadenas de medios de comunicación y demás grupos de interés que solicitan créditos y coberturas especiales como industrias en problemas.

b) Las organizaciones campesinas y propietarios rurales que demandan mayores subsidios para su operación, aun cuando no siempre se privilegie la eficiencia.

c) Las adquisiciones públicas diversas que no se sujetan a criterios de ahorro y eficiencia. Este tipo de gasto tiene identificados a sus benefactores; la teoría económica califica a este patrón de conducta como crony capitalism.

d) Los enormes pasivos laborales que tienen empresas para estatales como la Comisión Federal de Electricidad, Pemex, el Instituto Mexicano del Seguro Social (IMSS), entre otros. Cargan sobre estas instituciones un lastre de ineficiencia, rezago burocrático y pobre modernización, entrañando elevados costos de manutención.

e) Los rescates de 1995 a la banca mexicana a través del Fondo Bancario de Protección al Ahorro (FOBAPROA) y demás beneficiarios. En su momento, las autoridades justificaron estas acciones con el argumento de salvar empresas e instituciones estratégicas y de alto valor agregado en cuanto a empleos.

En el caso de México, la deuda pública sigue una inercia de crecimiento difícil de detener en vista de las necesidades de dichos recursos en términos de inversión y gasto corriente. Las acciones de recortes de gasto público se han aplicado de acuerdo con la ortodoxia económica. En los mismos se han 
implementado programas de ajuste y austeridad, afectando también a la clase media, pues la misma se ve precisada a pagar por servicios privados en educación y salud, aun cuando pueda tratarse de derechohabientes.

Asimismo, debe precisarse que en la mayoría de los países no existen los estabilizadores automáticos como los subsidios, créditos fiscales y apoyos en caso de desempleo, utilizados para aminorar los efectos de tales medidas. La evidencia revela que las políticas de ajuste aumentan el desempleo y reducen la inversión y recaudación (Parkin, 2014; Blanchard, 2017).

Cabe señalar que los incrementos en los tipos de interés y el tipo de cambio colocan mayor presión a la deuda contraída.

De acuerdo con Millán (2017), la peor combinación para la economía de un país son factores tales como su elevado endeudamiento, tasa cero de crecimiento, bajos ingresos, elevada inflación, desequilibrios financieros en sus cuentas externas, además de inestabilidad en sus variables macroeconómicas fundamentales. El autor refiere que en la década de los ochenta del siglo pasado la apuesta del gobierno por el petróleo elevó considerablemente el gasto público y ello colocó al país al borde del colapso.

Según Millán (2017), las causas fueron esencialmente:1) elvencimientode los bonos gubernamentales, predominantemente en manos de extranjeros; 2) salida de capitales; 3) reducción de las reservas internacionales; 4) desequilibrios en las cuentas fiscales; 5) depreciación del peso y, 6) la banca al borde de la quiebra. Ante esta situación, el gobierno mexicano solicitó una línea de crédito al gobierno de EU y de otros organismos internacionales. Este rescate financiero significó que el país aplicara un estricto programa de disciplina fiscal, ajuste al gasto y la venta de activos y empresas estatales.

La deuda pública de México tiende a crecer constantemente según se observa en la gráfica 2. Esta contempla todos los pasivos internos y externos del gobierno, el rescate bancario y carretero, así como la deuda de las empresas estatales, pensiones, además de otros conceptos. Estos pasivos aparentemente estaban bajo control; sin embargo, su crecimiento ha continuado y ahora está implicando que las calificadoras de riesgo alerten sobre sus niveles de mayores peligros. En el caso de México, el porcentaje de la deuda respecto al PIB es del 54.1 por ciento en el año 2020. Este tiende a incrementarse, reduciéndose el margen para que el gobierno actúe sin incrementar impuestos, crear nuevos cargos, o bien, reducir el gasto, extendiendo el estancamiento de la economía. 


\section{Gráfica No. 2. Evolución de la deuda pública de México en el periodo 1990-2020}

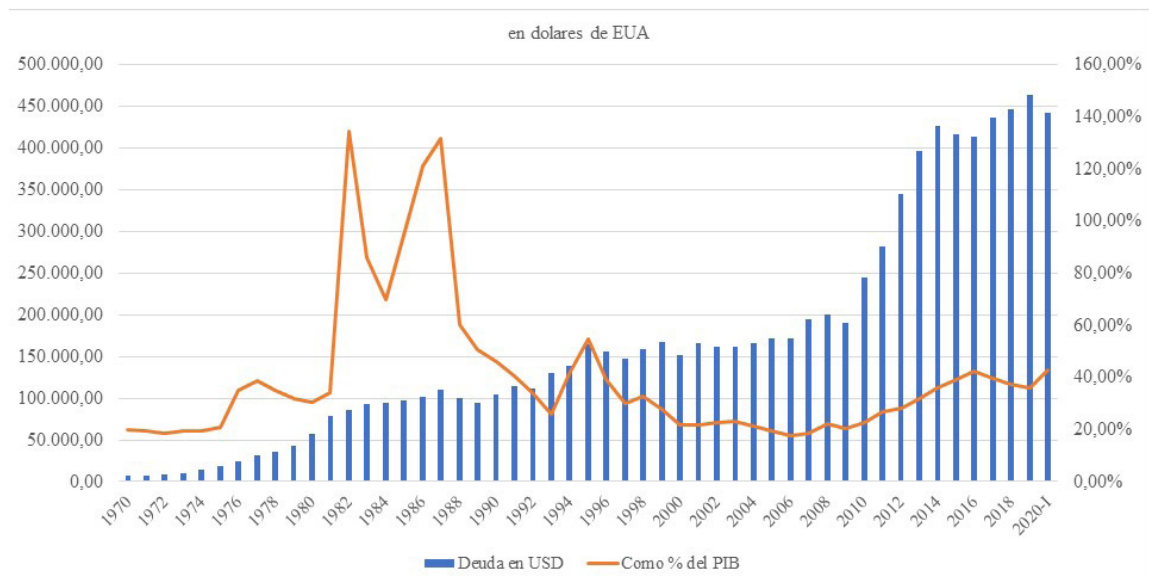

Fuente: Elaborado por los autores con base en los datos de Secretaria de Hacienda y Crédito Público (SHCP) (2020).

\subsection{El caso de la deuda de las entidades federativas}

A nivel subnacional hay entidades que tienen elevados niveles de deuda mayor al 100 por ciento, sobresaliendo Coahuila, Chihuahua, Nuevo Leon y Quintana Roo. También se encuentran aquellas que tienen tasas de endeudamiento consideradas sostenibles y con indicador de rango bajo, entre las que se encuentran Aguascalientes, Guanajuato, Guerrero, Queretaro, entre otras. Estas últimas tienen un futuro sin graves presiones y compromisos que se desprenden de las decisiones de no incurrir en deuda y déficit.

De acuerdo con el Centro de Estudios de las Finanzas Públicas (2020), los gobiernos de las entidades federativas participan con 85 por ciento de la deuda contratada, los municipios con 6.9 por ciento, los entes públicos estatales con 6.9 por ciento y los entes públicos municipales con 0.5 por ciento (vease cuadro 2). El mismo documento señala que la deuda se ha incrementado en 450 mil 379.2 millones de pesos en términos nominales, al pasar de 147 mil 412.4 millones de pesos al cierre de 2005 a 597 mil 791.6 millones de pesos al primer trimestre de 2020. 
Oshiel Martínez Chapa, Jorge Eduardo Salazar Castillo y Saul Roberto Quispe Aruquipa Deuda pública en México: dinámica e implicaciones de política

\section{Cuadro No. 2. Deuda estatal al tercer trimestre de 2020}

\begin{tabular}{|l|c|c|c|l|c|c|c|}
\hline Entidad & $\begin{array}{l}\text { Nivel de } \\
\text { deuda* }\end{array}$ & Rango & $(\%)$ & Entidad & $\begin{array}{l}\text { Nivel de } \\
\text { deuda }^{*}\end{array}$ & Rango & (\%) \\
\hline Aguascalientes & $\mathrm{S}$ & bajo & 27.1 & Morelos & $\mathrm{S}$ & Bajo & 59.1 \\
\hline Baja California & $\mathrm{S}$ & bajo & 49.9 & Nayarit & $\mathrm{S}$ & bajo & 80.6 \\
\hline $\begin{array}{l}\text { Baja California } \\
\text { S }\end{array}$ & $\mathrm{S}$ & bajo & 26.9 & Nuevo León & EO & medio & 108.2 \\
\hline Campeche & $\mathrm{S}$ & bajo & 29.4 & Oaxaca & $\mathrm{S}$ & bajo & 69.2 \\
\hline Coahuila & $\mathrm{EO}$ & medio & 153.6 & Puebla & $\mathrm{S}$ & bajo & 35.9 \\
\hline Colima & $\mathrm{S}$ & bajo & 64.4 & Querétaro & $\mathrm{S}$ & bajo & 2.30 \\
\hline Chiapas & $\mathrm{S}$ & bajo & 68.7 & $\begin{array}{l}\text { Quintana } \\
\text { Roo }\end{array}$ & $\mathrm{EO}$ & medio & 121.9 \\
\hline Chihuahua & $\mathrm{EO}$ & medio & 148.0 & $\begin{array}{l}\text { San Luis } \\
\text { Potosí }\end{array}$ & $\mathrm{S}$ & bajo & 27.6 \\
\hline CDMX & $\mathrm{S}$ & bajo & 49.8 & Sinaloa & $\mathrm{S}$ & bajo & 25.7 \\
\hline Durango & $\mathrm{S}$ & bajo & 85.5 & Sonora & $\mathrm{S}$ & bajo & 88.3 \\
\hline Guanajuato & $\mathrm{S}$ & bajo & 20.5 & Tabasco & $\mathrm{S}$ & bajo & 24.7 \\
\hline Guerrero & $\mathrm{S}$ & bajo & 23.5 & Tamaulipas & $\mathrm{S}$ & bajo & 57.1 \\
\hline Hidalgo & $\mathrm{S}$ & bajo & 28.8 & Veracruz & $\mathrm{S}$ & bajo & 83.8 \\
\hline Jalisco & $\mathrm{S}$ & bajo & 37.3 & Yucatán & $\mathrm{S}$ & bajo & 26.9 \\
\hline México & $\mathrm{S}$ & bajo & 41.0 & Zacatecas & $\mathrm{S}$ & bajo & 69.4 \\
\hline Michoacán & $\mathrm{S}$ & bajo & 61.2 & Promedio & $\mathrm{S}$ & bajo & 57.9 \\
\hline
\end{tabular}

Fuente: Flores (2020). *S (Sostenible); EO (En Observación)

Nota: Tlaxcala no es objeto de medición del sistema de alertas, toda vez que no cuenta con financiamientos y obligaciones inscritas en el Registro Público Único.

El crecimiento de la economía de México se ha situado lejos de las metas oficiales y ello ha tenido consecuencias adversas. El crecimiento real es casi de la mitad del proyectado, lo cual contribuye al mismo crecimiento de la informalidad laboral del 60 por ciento en promedio. El país se beneficiaba anteriormente con ingresos fiscales derivados de las exportaciones petroleras y de los altos precios del crudo. Ello llegó a representar casi una tercera parte de los ingresos públicos. Sin embargo, ahora se están importando más productos petrolíferos y los ingresos públicos por este concepto se han reducido. Los gobiernos anteriores intentaron compensar esta reducción de ingresos con mayores impuestos y nuevos cargos, lo cual 
provocó malestar social.

Desafortunadamente, la coyuntura deingresos petroleros extraordinarios del periodo 2010-2014 dio lugar al derroche e irresponsabilidad pública, pues buena parte de dichos recursos provocaron alzas en los niveles de deuda de las entidades del país y también en las dependencias públicas. Existen otras razones que han propiciado el incremento de la deuda en el país, las cuales han motivado que se ha denunciado por parte de ciudadanos y partidos de oposición, justo el gobierno actual (2018-2024) busca contener.

La normatividad inadecuada en el manejo de los recursos ha dado lugar a que en las entidades federativas y municipios los funcionarios abusen. A mayor cantidad de recursos federales otorgados, le corresponde mayor nivel de deuda estatal y municipal. A fin de poner orden en las finanzas públicas y debido a las presiones de la sociedad civil, el Congreso de la Unión aprobó la Ley de Disciplina Financiera de las Entidades Federativas y Municipios en 2018. Con esta legislación se pretende que en lo sucesivo se haga pública información relevante como el monto de préstamos, tasa de interés, condiciones, plazos, garantías, obligaciones, así como la utilización de los fondos de dichos recursos. Dicha ley requiere de plena licitación en la contratación de créditos, además de que debe ser aprobada por dos terceras partes de los congresos estatales y municipales, respectivamente.

La situación de endeudamiento público en un contexto de bajo crecimiento económico ha tenido consecuencias, pues las políticas de recorte del gasto público recetadas por las autoridades monetarias internacionales y por los gobiernos nacionales han agravado la situación. En el caso de México, la extendida austeridad del presente significa menores recursos para inversión en infraestructura, educación, salud y demás rubros que requieren atención urgente. Los programas de austeridad significan un menor dinamismo en la economía.

Los márgenes de maniobra que tiene el gobierno de México en la coyuntura actual de recesión son: 1) crear nuevos impuestos; 2) incrementar los ya existentes; 3) una combinación de los dos anteriores; 4) reducir el gasto público y, 5) incrementar deuda. Debe señalarse que estas son acciones que se han implementado en el pasado y no han mejorado la situación del grueso de la población. Sin embargo, siguen siendo una tentación en el presente. No obstante, son política y electoralmente costosas; tampoco son bien recibidas en virtud de los reclamos de la sociedad respecto a la discrecionalidad de las políticas de gasto excesivo del pasado. Ahora la sociedad civil exige mayor transparencia y rendición de cuentas, asi como limitar el gasto innecesario en la asignación de los recursos públicos.

Como factores adicionales que guardan una estrecha corresponsabilidad con el incremento del gasto público deben incluirse las enormes percepciones de los altos funcionarios del poder judicial, los sueldos de los consejeros 
electorales, diputados y senadores. Debe enfatizarse que no son reguladas por autoridad alguna, es decir, estos funcionarios tienen soberana facultad para determinar los incrementos que estimen necesarias a sus percepciones. La crisis institucional y económica que atraviesa México ha elevado las críticas de la sociedad civil a fin de limitar este tipo de excesos. El actual gobierno (2018-2024) ha propuesto colocar límites a las percepciones de los funcionarios públicos.

Siguiendo con este mismo orden de ideas, el financiamiento público al Instituto Nacional Electoral (INE) advierte un crecimiento constante. En esta institución no se privilegian las economías de escala debidas fundamentalmente a la especialización, a la acumulación de la experiencia, al empleo de la tecnología y, en general, a una lógica de eficiencia como la que se llevan a cabo en las empresas privadas exitosas. El INE organiza los procesos electorales en México a partir de la creación de un marco legal en el que se establece el tiempo de inicio y finalización de las campañas políticas, el sistema de registro, sanciones y arbitraje a los actores político-electorales. Sin embargo, se cuestiona, entre otras cosas, lo extraordinariamente costosa que resultan las elecciones en el país.

De acuerdo con Heath (2015), México ha implementado una reforma fiscal recaudatoria junto con medidas de fiscalización casi extremas. El gobierno ha logrado un incremento importante en sus ingresos, aunque ello no se ha aprovechado para efectos de consolidación fiscal con el fin de sanear las finanzas. Los gobiernos impulsaron políticas de mayor gasto: el incremento extraordinario en el gasto que ha conducido a déficit fiscal como porcentaje del PIB más elevado hasta ahora. Para Heath, el gasto ha perdido su eficacia y no contribuye al crecimiento económico del país. El gobierno dispone de estos recursos que deben también apoyar a empresas y a los hogares y así crear empleos. De otro modo, al no considerarlo, se genera insatisfacción social. En ese sentido, el gobierno debe gastar menos, pero de mejor forma. Se trata de que la economía crezca y así se reduzca la pobreza.

\section{Métodos y resultados}

Con datos del Banco Mundial (2020) respecto a la variable deuda pública de México y como parte de la metodología propuesta para esta investigación se utiliza la técnica econométrica del Análisis de Regresión:

$$
\boldsymbol{Y}=\boldsymbol{\beta}_{o}+\boldsymbol{\beta}_{1} \boldsymbol{X}+\boldsymbol{\varepsilon}
$$


Donde:

$Y=$ Variable dependiente $\mathrm{o}$ de respuesta

$X=$ variable independiente (predictora o explicativa)

$\beta_{o}=$ ordenada al origen (valor de $\mathrm{Y}$, cuando $\mathrm{X}$ es igual a o)

$\beta_{1}=$ pendiente de la recta de regresión

$\varepsilon=$ término de error aleatorio

En el modelo en cuestión se estiman, a partir de una muestra, los valores verdaderos, los cuales son:

$$
\boldsymbol{y}=\boldsymbol{b}_{o}+\boldsymbol{b}_{1} \boldsymbol{X}
$$

En este caso, $\boldsymbol{y}$ es el valor pronosticado de Y.

En el ejercicio relativo a la tasa de crecimiento de la deuda gubernamental de México expresada en dólares lo que se plantea es lo siguiente:

$$
Y_{t}=Y_{o}(1+r)^{t}
$$

Donde:

$Y_{o}=$ es el periodo inicial o el principio de $Y$

$Y_{\mathrm{t}}=$ es el valor del tiempo, o $t$

$r=$ es la tasa de crecimiento de $Y$

Una vez que se manipula la ecuación de crecimiento, y toma el valor en logaritmo natural, lo que se tiene es:

$$
\ln Y_{t}=\ln Y_{o}+t \ln (1+r)
$$

Sin embargo, por tratarse de un modelo de crecimiento semilog, lo que se pretende es calcular dicho crecimiento con solo la variable dependiente $Y$, de manera que la variable independiente, en este caso $\mathrm{X}$, toma el valor de tiempo: 1, 2, 3, 4, etcétera (Gujarati y Porter, 2010). En el ejercicio propuesto se calculan las tasas de crecimiento de la deuda de México para el periodo 1990-2020. 


\section{Cuadro No. 3. Mínimos Cuadrados Ordinarios usando las observaciones 1990-2020 $(\mathrm{T}=\mathbf{3 1})$}

\begin{tabular}{|l|c|c|c|c|c|c|}
\hline \multicolumn{7}{|c|}{ Variable dependiente: In Deuda México en USD } \\
\hline & Coeficiente & Desv. Típica & Estadístico $t$ & valor $p$ & & R-cuadrado \\
\hline Constante & 11.041427 & 0.09537451 & 115.769 & $3.2689 \mathrm{E}-40$ & $* * *$ & \\
\hline Tiempo & 0.0303140 & 0.00520311 & 5.826 & 0.00000256 & $* * *$ & 0.5392718 \\
\hline
\end{tabular}

Fuente: resultados de la aplicación del Modelo de regresión de MCO utilizando el programa econométrico GRETL

\subsection{Presentación e interpretación de los resultados}

Del modelo de regresión estimado se deriva lo siguiente. En el período estudiado el cambio en el valor de la variable Deuda, en función del Tiempo, es muy significativo ( $\mathrm{p}<0.00001)$. El intervalo de confianza para el coeficiente de regresión del Tiempo, con un nivel $\alpha=0.05$ es (0.0196, 0.0409); es decir, siempre creciente al aumentar el valor de la variable Tiempo, y como dicho intervalo de confianza excluye el valor o, se rechaza la hipótesis nula:

Ho: La deuda pública no ha evolucionado en el mediano y largo plazo. Por lo que se considera que los datos son congruentes con la hipótesis alterna: Ha: La deuda pública sí ha evolucionado en el mediano y largo plazo.

Tomada la hipótesis alterna, al observar los valores positivos de los extremos del intervalo de confianza para la variable Tiempo, se puede especificar que la deuda pública, en México, sí ha evolucionado y lo ha hecho con una pendiente de regresión positiva; es decir tiende a crecer a través del periodo estudiado.

En el subconjunto de datos en el período 1990-2008 un intervalo de confianza del 95\% para el coeficiente de regresión del Tiempo es (-0.0237, -0.0001) con un $\mathrm{R}^{2}=0.2124$ y una probabilidad del estadístico de la pendiente de regresión de 0.047. En contraste el subconjunto de datos en el período 2009-2020 un intervalo de confianza del 95\% para el coeficiente de regresión del Tiempo es (0.0770, 0.0960), con un $\mathrm{R}^{2}=0.9762$ y una probabilidad del estadístico de la pendiente de regresión de 1.8909E-o9.

Aun cuando son de diferente signo las pendientes de las rectas de regresión en cada subconjunto muestral, en ambos casos también se rechaza la hipótesis nula probada anteriormente; siendo en el segundo subconjunto más significativa estadísticamente la relación entre las variables estudiadas. Por lo que el modelo propuesto es apropiado para 
fines de pronóstico, particularmente a partir de los datos más recientes. En la figura No. 3 se observa el pronóstico calculado para los próximos 5 años; como es de esperar el intervalo de confianza se amplía, al alejarse hacia el futuro, de los valores de la muestra estudiada.

\section{Figura No. 3 Pronóstico de crecimiento de la deuda pública de México}

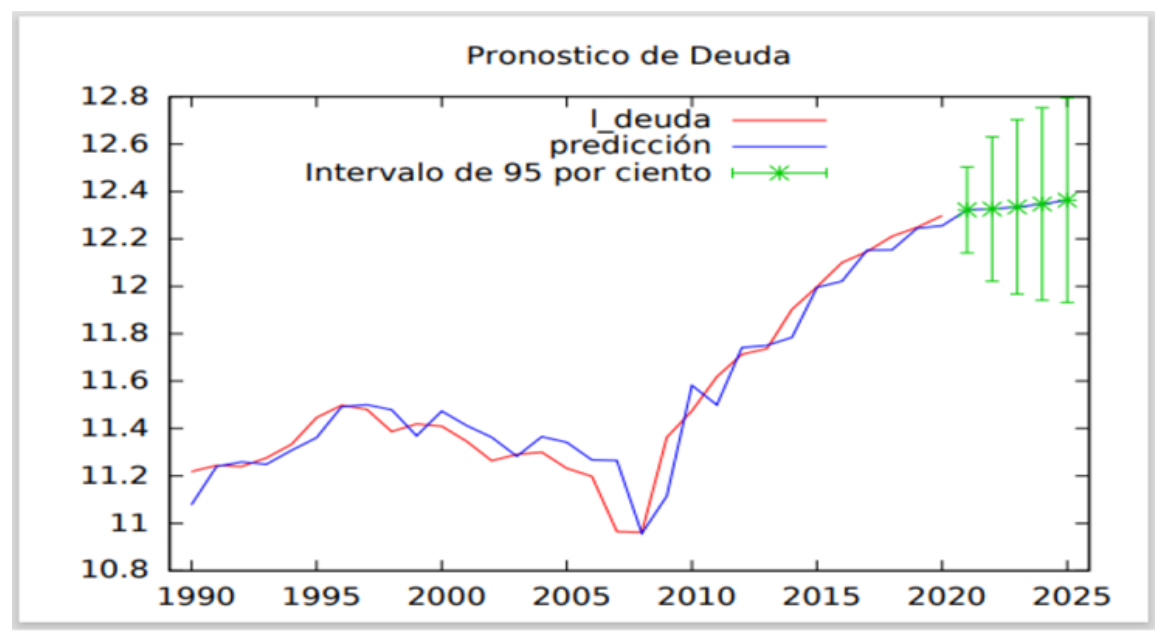

Fuente: Elaborado por los autores en base a programa econométrico GRETL.

\section{Conclusiones y recomendaciones}

Al hablar específicamente de la deuda de las naciones, debe tomarse en cuenta que el crecimiento de esta se ha extendido más allá de lo que lo hecho la producción. Su crecimiento es superior a las de los salarios y las percepciones del grueso de la población económicamente activa. Dicha deuda se explica, en buena parte, por razones como el gasto extraordinario que significa un creciente Estado de bienestar, la cultura de irresponsabilidad en el manejo de las finanzas públicas, así como el hecho de que los funcionarios encargados de las finanzas públicas han actuado por mucho tiempo con discrecionalidad y opacidad, sin consecuencias para ellos como la inhabilitación de cargos públicos, la obligación de resarcir el daño al patrimonio de la nación, entre otras medidas.

Con base en lo aquí expresado, México enfrenta limitantes serias en rubros tan fundamentales como la falta de disciplina fiscal y monetaria, al 
alcanzar los actuales niveles de deuda de más de 50 por ciento. Esta situación es el resultado de no actuar a tiempo en lo que respecta a disminuir el gasto excesivo. La disminución en la inversión, además de la prevalencia de un entorno internacional recesivo, han afectado el desempeño de la economía mexicana, lo cual se traduce en una menor recaudación gubernamental. Para recomponer esta situación hay que combatir, adicionalmente, otros lastres que se han arraigado, como la corrupción e ineficacia las cuales obstruyen la aplicación del Estado de derecho.

No son pocos quienes se preguntan cómo es que la clase política es dada a gastarse enormes recursos del erario. Esta propensión al derroche ha significado el enojo de la ciudadanía. Sin embargo, aumentar cargos, crear nuevos impuestos, así como reducir beneficios sociales son acciones que se hallan al alcance del gobierno, sin embargo, ello va en detrimento del bienestar de la mayoría de los ciudadanos. La ciencia política y la economía convergen en los asuntos públicos y sociales bajo la premisa fundamental de que los ciudadanos (votantes y contribuyentes) tienen como objetivo maximizar su beneficio. Entre los diversos retos del actual gobierno en México se hallan la reducción del costo económico de los procesos electorales y asi dejar mejores resultados a la sociedad.

El gasto público debe incidir en el crecimiento de la economía y en la reducción de la pobreza. Empero, en México se destina un presupuesto mayor para gasto corriente que para gasto de inversión. Mayor tamaño del gobierno no necesariamente se traduce en bienestar para la población de escasos recursos. Se requiere, por tanto, un Estado menos costoso, más ágil, además de que sea promotor del progreso.

Otra exigencia ciudadana tiene que ver con la necesidad de acotar la desmedida discrecionalidad en el uso del gasto público, sin que ello implique que las políticas de austeridad conlleven a un mayor deterioro en la infraestructura física y social expresada en recortes en las asignaciones presupuestarias de escuelas, hospitales, carreteras, aeropuertos, parques y demás.

Por mucho tiempo la clase política y gubernamental ha sacado provecho de su posición de ventaja, para lo cual ha creado mecanismos de intervención a partir de esquemas considerados como de "interés público" o "interés nacional", con lo cual se restringen oportunidades de que otros agentes sociales y económicos expongan sus puntos de vista, además de que tengan la información sobre las implicaciones del gasto público.

En base a lo anteriormente expresado, el gobierno está llamado a actuar con mayor sentido de responsabilidad en el manejo de los recursos públicos, pues se trata de los ciudadanos que los sostienen con sus impuestos y cargos. Ahora se propone que el gobierno sea eficaz y promotor del bienestar, además de respetuoso de las libertades sociales. Los gobiernos 
con facultades discrecionalidades no han privilegiado los principios de respeto y apego a las leyes de protección ciudadana.

\section{Referencias Bibliográficas}

AYHAN KOSE, M; NAGLE, Peter; OFHNORGE, Franziska; SUGAWARA, Naotaka. 2020. "Global waves of debt: Causes and consequences" In: World Bank. Disponible en línea. En: https://www.worldbank.org/en/ research/publication/waves-of-debt. Fecha de consulta: 14/012/2021.

BANCO MUNDIAL. 2020. Total del servicio de la deuda (\% del PIB). Disponible en línea. En: https://datos.bancomundial.org/indicador/DT.TDS. DECT.GN.ZS. Fecha de consulta: 27/11/2020.

BLANCHARD, Olivier. 2017. Macroeconomía. Pearson. Ciudad de México, México.

BUCHANAN, James. 2000. "Democracia limitada o ilimitada" En: Estudios Públicos. Disponible en línea. En: https://lyd.org/wp-content/ uploads/2012/04/buchanan-Democracia-Limitada-o-ilimatada.pdf. Fecha de consulta: 12/04/2020.

CAMARA DE DIPUTADOS DEL HONORABLE CONGRESO DE LA UNIÓN. 2018. la Ley de Disciplina Financiera de las Entidades Federativas y Municipios. Disponible en línea. En: http://www.diputados.gob.mx/ LeyesBiblio/pdf/LDFEFM_300118.pdf. Fecha de consulta:07/05/2020.

DICEY, Albert Venn. 2008. "Lectures on the relation between law and public opinion in England during the Nineteenth century" En: Liberty Fund, Indianapolis. Disponible en línea. En: https://oll.libertyfund.org/ pages/dicey-on-law-and-public-opinion-in-the-19th-century. Fecha de consulta: 24/04/2021.

DORNBUSCH, Rudiger; FISCHER, Stanley; STARTZ, Richard. 2002. Macroeconomía. McGraw Hill. Ciudad de México, México.

FONDO MONETARIO INTERNACIONAL. 2020. Historical Public Debt Database y Maddison Database Project. Disponible en línea. En. https:// www.imf.org/en/Publications/FM/Issues/2020/o9/30/october-2020fiscal-monitor. Fecha de consulta: 23/05/2021

FLORES, Efrén. 2020. "La deuda de estados toca récord en 2019. La SHCP ve más riesgos en Coahuila, Chihuahua, NL y Quintana Roo". En: Sin Embargo. Disponible en línea. En: https://www.sinembargo.mx/o5-012020/3704725. Fecha de consulta: 15/01/2020. 
FRIEDMAN, Rose. 1989. "La corriente en los asuntos de los hombres". En: Revista Libertas. Vol. 11. No. 11, pp. 1-15. Disponible en línea. En: https://www.eseade.edu.ar/files/Libertas/39_3_Friedman.pdf. Fecha de consulta: 24/04/2021.

HAYEK, Frederich Von. 2017. Camino de servidumbre. Unión Editorial. Disponible en línea. En: https://www.elcato.org/sites/default/files/ camino-de-servidumbre-libro-electronico.pdf Fecha de consulta: 03/02/2020.

HEATH, Jonathan. 2015. "La eficiencia del gasto público". En: Alto Nivel. Pp. 22-25. Ciudad de México, México.

HEILBRONER, Robert; MILBERG, William. 1999. "La evolución de la sociedad económica”. Prentice Hall. Naucalpan de Juárez, México.

GUJARATI, Damodar N; PORTER, Dawn C. 2010. "Econometría”. McGraw Hill. Ciudad de México, México.

KRAUSE, Martin. 2014. El Faro y el Bazar: Economía, instituciones y políticas públicas. Editorial Francisco Marroquín. Disponible en línea. En: https://www.researchgate.net/publication/273425390_El_Foro_y_ el_Bazar_Economia_Instituciones_y_Politicas_Publicas. Fecha de consulta: 16/01/2021.

MÉNDEZ, Ana; MORALES, Wilmer. 2000. "Globalización en América latina: ajustes, desajustes y perspectivas” En: Cuestiones Políticas. Vol. 16, No. 24, pp. 16-24.

MILLÁN, Julio. 2017. Deuda: peligro para México. Alto nivel. Ciudad de México, México.

ORGANIZACIÓN PARA LA COOPERACIÓN Y EN DESARROLLO ECONÓMICO. 2009. Respuesta estratégica de la OCDE a la crisis económica y financiera. Disponible en línea. En: https://www.oecd.org/ centrodemexico/medios/42981787.pdf. Fecha de consulta: 7/05/2021.

PARKIN, Michael. 2014. Economía. Pearson. Ciudad de México, México.

ROPKE, William.1960. "Estado benefactor e inflación crónica" En: Fundación Hacer. Disponible en línea. En: http://www.hacer.org/pdf/Ropkeo1. pdf. Fecha de consulta: 19/06/2021.

SAMUELSON, Paul; NORDAHUS, William. 2010. Economía con aplicaciones a América Latina. McGraw Hill. Ciudad de México, México. 
SECRETARÍA DE HACIENDA Y CRÉDITO PÚBLICO. 2020. Informes sobre la situación económica, las finanzas y la deuda públicas al segundo trimestre de 2020. Disponible en línea. En: https://www.gob.mx/shcp/ prensa/comunicado-no-062-informes-sobre-la-situacion-economicalas-finanzas-publicas-y-la-deuda-publica-al-segundo-trimestre-de2020?state=published. Fecha de consulta: 02/11/2020.

WORLD POPULATION REVIEW. s/f. "Deuda como porcentaje del PIB". Disponible en línea. En: https://worldpopulationreview.com/countries/ countries-by-national-debt. Fecha de consulta: 02/06/2021. 

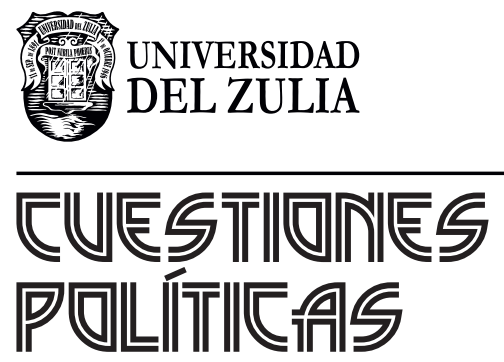

Vol. 39 N $^{\circ} 71$

Esta revista fue editada en formato digital y publicada en diciembre de 2021, por el Fondo Editorial Serbiluz, Universidad del Zulia. Maracaibo-Venezuela 